\title{
Detection of Honeybee Venom in Envenomed Tissues by Direct MALDI MSI
}

\author{
Simona Francese, ${ }^{\text {a }}$ Duccio Lambardi, ${ }^{\mathrm{b}}$ Guido Mastrobuoni, ${ }^{\text {a }}$ \\ Giancarlo la Marca, ${ }^{\mathrm{c}}$ Gloriano Moneti, ${ }^{\mathrm{a}}$ and Stefano Turillazzi ${ }^{\mathrm{b}}$ \\ ${ }^{a}$ Interdepartmental Centre of Mass Spectrometry, University of Florence, Florence, Italy \\ ${ }^{\mathrm{b}}$ Department of Evolutionary Biology, University of Florence, Florence, Italy \\ ${ }^{c}$ Department of Pharmacology, University of Florence, Mass Spectrometry Laboratory, Metabolic and \\ Neuromuscular Unit, Meyer Children's Hospital, Florence, Italy
}

\begin{abstract}
A new analytical approach using matrix-assisted laser desorption/ionization mass spectrometry imaging (MALDI MSI) for the study of honeybee venom is shown. In vitro and in vivo models simulating the bee sting have been developed using live honeybees and, as the envenomation sites, pig ears and rat legs; MALDI MSI has been used to map, over time, the diffusion and distribution of three venom allergens (Api m 1, Api m 4, and Api m 6) and two venom toxins (apamine and mast cell degranulating peptide). In conjunction with other classical biochemical techniques and high resolution mass spectrometry (HRMS), structural data have been obtained that contribute to current understanding of honeybee venom composition. Initial data have also been obtained demonstrating the feasibility of mapping the organism's response to the sting. The opportunity to monitor venom diffusion and the organism's response at the same time might open new pathways for in vivo preclinical studies in designing and testing new venom immunotherapy (VIT). (J Am Soc Mass Spectrom 2008, xx, xxx) @ 2008 Published by Elsevier Inc. on behalf of American Society for Mass Spectrometry
\end{abstract}

$\mathrm{T}$ The study of hymenoptera venom is important, as it can trigger allergic reactions in humans that can be fatal. In particular, venom from European honeybees (Apis mellifera) is reported to be the best characterized of all insect venoms [1, 2]; nonetheless, current literature demonstrates that this topic is currently of widespread scientific interest. When threatened, honeybees deliver the venom through a peculiar barbed sting designed to remain embedded in the victim's flesh [3]. Axial movements of the two barbed stylets causes further burying of the sting over a period of $\sim 30 \mathrm{~s}$ [4]. Once the sting is fully embedded, the venom discharge starts. Honeybee venom (HBV) is composed of a wide spectrum of biomolecules, ranging from biogenic amines to peptides and proteins, whose structure and function have been determined [5-8]; full characterization of venom components, including function, synergistic mechanisms in the toxic reaction, crossreactivity, and the range of biomolecules released upon envenomation, is crucial for the elucidation of $\mathrm{HBV}$ action and therefore currently under investigation by a number of research groups [9-11]. The disclosure of this information would have considerable impact on current venom immunotherapy (VIT) by allowing tailoring of the therapeutic treatment of an individual's allergic reaction. In 2005, Peiren and coworkers re-

Address reprint requests to Dr. S. Francese, Department of Health and Wellbeing, Biomedical Research Center (BMRC), Sheffield Hallam University, Howard Street, S1 1WB, Sheffield, UK. E-mail: s.francese@shu.ac.uk ported that VIT with recombinant HBV allergens, assayed in stage I clinical trials, was more specific and safer than treatments employing bee-derived venom [9]. A full venom characterization would also help to possibly improve current HBV based therapeutics, reported in particular for arthritic and rheumatic conditions [12].

The reaction to HBV (and to hymenoptera in general) can be of an allergic and/or toxic nature. Allergens are generally proteins (enzymes) and may trigger life threatening and sometimes fatal IgE-mediated anaphylactic reactions. Toxins are generally peptides and cause localized reactions. An exception to these groupings can be made for melittin, which is a peptidic allergen.

The regulation of IgE synthesis is mediated by the release of small molecules like histamine, sulfidoleukotrienes (LTC4, LDT4, LTE4, LTA4) and proteins such as cytokines. Th-2 profile cells produce interleukin-13 and interleukin-4, which promote IgE synthesis by B cells, whereas Th- 1 cells produce IFN- $\gamma$, which prevents hypersensitivity by playing a negative regulatory role in the development of Th2 cells [13]. Diagnosis of IgE-mediated insect venom allergy by classical allergenspecific IgE tests is often not reliable; tests detecting release of sulfidoleukotrienes such as the CAST-ELISA may therefore be a valid alternative [14].

The International Union of Immunological Societies (IUIS) reports the list of honeybee venom allergens updated to the 28th of January 2008 (http://www. allergen.org/Allergen.aspx). There are six officially rec- 
ognized venom allergens: phospholipase A2 (Api m 1), hyaluronidase (Api m 2), acid phosphatase (Api m 3), melittin (Api $\mathrm{m} \mathrm{4}$ ), a newly discovered small protein Api $\mathrm{m} \mathrm{6}$, and the CUB serine protease (Api $\mathrm{m}$ 7).

Api $\mathrm{m} 1$ is the most characterized honeybee venom allergen and is reported to account for $10 \%$ to $12 \%$ of crude venom [12]. The primary amino sequence of the mature protein has a MW of $\sim 15.2 \mathrm{kDa}$. The enzyme is also present in glycosylated isoforms, which are not yet extensively characterized for their microheterogeneity [15]. Nevertheless, it is known that the glycostructures are $\mathrm{N}$-linked to asparagine 13 [16], giving rise to higher molecular weight species. The allergenic actions of Api $\mathrm{m} 1$ are a result of scavenging actions on biological membranes, disrupting the main conformation and producing lysophospholipids as well as long-chain anionic fatty acids. Many studies have reported a synergistic activity with melittin, where the latter would open membrane channels leading to the exposure of the phospholipid catalytic site of Api m 1 [3, 12].

Api $\mathrm{m} 2$ is also a very important allergen accounting for $1 \%$ to $2 \%$ of the crude venom [3]. Its molecular weight ranges from $40.7 \mathrm{kDa}$ (primary amino acidic sequence) to $60 \mathrm{kDa}$ [13], depending on the carbohydrate content $\mathrm{N}$-linked to the protein. Api $\mathrm{m} 2$ is called the "spreading factor" because it disrupts hyaluronic acid connective tissue matrix allowing other allergens and toxins to infiltrate and spread in the tissue [3]. Api $\mathrm{m} 3$ is a glycoprotein accounting for $1 \%$ to $2 \%$ of the venom dry weight $[3,13]$. In 2005, this protein was reported by Peiren and coworkers [9] to have been removed from the list of allergens because of incomplete IgE binding and lack of satisfactory sequence data. Nonetheless, the IUIS has re-introduced this enzyme into the allergen list. This is probably due to the studies of Grunwald and coworkers [8] as well as Hoffman and collaborators $[17,18]$ who determined by several means its molecular weight $(43.9 \mathrm{kDa}$, processed primary amino acid sequence) and its $\operatorname{IgE}$ binding capacity as a major allergen. Although the biological mechanism of action is not fully elucidated, the deduced amino acid sequence for Api $\mathrm{m} 3$ exhibits several features in common with high molecular weight phosphatases, which hydrolyze phosphomonoesters in a two step mechanism involving a histidine at the active site [8]. Api $\mathrm{m} 4$, a basic peptide of 26 residues $(\sim 2.8 \mathrm{kDa})$, is considered a minor allergen and accounts for $45 \%$ to $50 \%$ of the venom dry weight $[12,19]$. Api $\mathrm{m} 4$ exhibits an amphipathic structure; its polar and nonpolar ends allow it to interact with lipid membranes and ultimately increases permeability. In particular, its tetrameric structure has been suggested to act as an ionophoretic scavenger. This property would be responsible for skeletal muscle, heart, and blood vessel contraction as well as nerve depolarization, with the latter macroscopically translating into the intense pain caused by the sting. Api $\mathrm{m} 6$ was isolated and fully characterized for the first time in 2001 by Kettner and coworkers [20], who found it to bind to IgE antibodies in greater than
$40 \%$ to $42 \%$ of bee venom hypersensitive patients, thus making it a minor allergen [20,21]. Api $\mathrm{m} 6$ accounts for $1 \%$ to $2 \%$ of the crude venom, with four isoforms in equimolar amounts and with the same allergenicity [20]. The four isoforms, named Api m 6.01 to Api m 6.04, share a common central amino acid sequence of 67 residues and differ only in their amino and carboxyterminus by up to 6 amino acids. API $\mathrm{m} 6.01$ and Api $\mathrm{m}$ 6.03, with five disulphide bridges, have a molecular weight of $7179.6(5-71)$ and $7588.1 \mathrm{Da}(1-71)$. The isoforms, Api m 6.02 and 6.04 exhibit an extra two amino acids at the carboxy-terminus (leucine and proline, not necessarily in this order) so that their molecular weights are 7389.9 (5-73), and $7798.3 \mathrm{Da}$ (1-73). respectively [20]. Reasons for the presence of these isoforms have been described by Perein and collaborators. In their work, Api m 6 is shown to derive from a single locus, but the protein level variation is caused by it sitting near a misassembled section of the honeybee genome sequence. The authors proposed that a substantial number of indels at and near Api m 6 might be the reason for this misassembly. Although it is known to bind to IgE, a fundamental knowledge of its biological activity is still unknown. Api $\mathrm{m} 7$ is the last known bee venom protein allergen. It was first reported by Shkenderov et al. in 1973 [22] as an allergenic protein of $\sim 39$ $\mathrm{kDa}$. It was then detected by other laboratories [23-26], and shown to contain a typical tryptic protease domain with a serine protease catalytic triad. Despite not being reported in the official allergen list, the newly discovered HBV Icarapin (MW 19.6 kDa) [9] is a glycoprotein worth mentioning along with the other allergens, as it has been cloned, expressed and found to bind IgE [10].

Concerning venom bioactivity, it is important to also mention two peptides, which are not allergens but are considered major venom toxins: apamine and mast cell degranulating (MCD) peptide. Apamine is a small 18 amino acids ( $\mathrm{MW} \sim 2.0 \mathrm{kDa}$ ) well characterized peptide accounting for less than $2 \%$ of venom dry weight. Its neurotoxic action results from blocking the sodium and calcium post-synaptic ion channels and this, in turn, blocks many inhibitory or hyper-polarization effects, including $\alpha$-adrenergic, cholinergic, and purinergic pathways. In neurons of both vertebrates and invertebrates, the post-synaptic ion channels play an important role in repetitive activities, therefore apamine downstream action ultimately blocks these activities [12].

The mast cell degranulating (MCD) peptide is chemically very similar to apamine and in fact it was found to share the same $3^{\prime}$-exon [27]. It accounts for $2 \%$ of the venom dry weight $[3,12]$ and comprises 22 amino acid residues $(\mathrm{MW} \sim 2.6 \mathrm{kDa}$ ). At low concentrations, $\mathrm{MCD}$ causes mast cell break down, with a consequent massive release of histamine, thus contributing to the overall allergic reaction.

So far, researchers studying HBV have concentrated their efforts on elucidating the properties of the allergenic proteins and peptides [26, 28, 29], on their isolation and characterization [21], on VIT [30], and on the 
study of tissue damage from a physiological point of view [31]. The study, isolation and characterization of allergenic proteins and peptides are being performed using much more sophisticated analytical techniques including: gene cloning, mass spectrometry, X-ray and genomic techniques [21]. In this context, MALDI mass spectrometry has proven to be a powerful tool, especially when used in a typical bottom up proteomic approach [9].

Recently, a new mass spectrometry technique named matrix-assisted laser desorption/ionization mass spectrometry imaging (MALDI MSI) has shown great potential for mapping a wide range of biomolecules (peptides and proteins, drugs, lipids, metabolites) directly from frozen tissue sections without the need for antibodies or molecular probes [32-35]. Typically, a thin tissue section is cut on a cryostat and spray coated with an appropriate organic matrix. A raster of points is defined and the laser (typically $\mathrm{N}_{2}, \mathrm{Nd}$ :YAG) will fire on each of the points in the grid so that a set of mass spectra will be acquired (microprobe mode). Each mass spectrum, therefore, represents the local molecular composition at known $x, y$ coordinates. An ion image can be obtained by simply retrieving via software the $m / z$ of interest [Francese, S.; Dani, F. R.; Traldi, P.; Mastrobuoni, G.; Pieraccini, G.; Moneti, G. MALDI Imaging Mass Spectrometry, from Its Origin Up to Today: The State of the Art. CCHTS, in press]; the software will produce an ion image by plotting the intensity of a selected $\mathrm{m} / \mathrm{z}$ value as function of the sampling position so that each image pixel correlates to both a specific $\mathrm{m} / \mathrm{z}$ value and to the corresponding region of the original sample in which the analyte was detected. The ion intensity is shown in the image as a change in "brightness/hue" for each pixel.

Here, to the authors' knowledge, for the first time MALDI MSI has been applied to investigate the on tissue direct detection of HBV upon honeybee sting. In particular, three allergens (Api m 1, Api m 4, and Api m 6) and two neurotoxins (apamine and MCD) have been monitored. The opportunity to explore a wide dynamic molecular weight range allowed the authors to analyze both the peptide and the protein fractions of the venom, over different post-envenomation times. Specific protocols are needed and are under current development to map the distribution of the other allergens. Api m 2, Api m 3, and Api m 7 are, per se, much higher molecular weight biomolecules, to some extent glycosylated (Api $\mathrm{m} 2$, Api $\mathrm{m} 3$ ), and in the case of Api $\mathrm{m} 3$, also found as a dimer [36, 37]. Finally, they represent altogether not more than $5 \%$ to $6 \%$ of the honeybee venom, which adds a further level of difficulty in their detection on tissue.

An in vitro time course experiment has been designed, using live honeybees to sting a $2 \mathrm{~cm}^{2}$ piece of porcine skin (ear). Venom distribution was then mapped within a transverse section of the skin. Also, a similar in vivo experiment was carried out with live animals (rats), that is, the "victim" was alive at the time of the venom inoculation. Data obtained so far provide the opportunity to map not only venom diffusion but also the organism's biochemical response. This possibility might open a new path for in vivo clinical trials in designing and testing new VIT.

\section{Experimental}

\section{Materials}

Acetonitrile, trifluoroacetic acid (TFA) and formic acid were purchased from Mallinckrodt Baker (Milan, Italy). $\alpha$-Cyano-4-hydroxycinnamic acid ( $\alpha \mathrm{CHCA})$, ethanol, methanol, dithiothreitol, DPX mountant, and Harris's modified haematoxylin and eosin solution ( $\mathrm{H}$ and $\mathrm{E})$ were purchased from Sigma-Aldrich (Milan, Italy). Sinapinic acid was obtained from Fluka (Milan, Italy). Porcine ears were collected unsteamed from a local abattoir (Arezzo, Italy). Honeybees (Apis mellifera) were captured in the surroundings of the laboratory and kept at constant temperature and humidity conditions with sugary water ad libitum until used for venom extraction and sting experiments. Conductive ITO glass slides were obtained from Bruker Daltonics (Bremen, Germany). Rats were purchased from Harlan, Udine, Italy.

\section{Honeybee Venom Collection}

Venom from one honeybee was collected with a capillary by gently squeezing the venom sack. It was then diluted in $50 \mu \mathrm{L}$ of double distilled water. The sample was then centrifuged and the supernatant recovered and dried in a vacuum centrifuge (Thermo Fisher, San Jose, CA). The sample was reconstituted in $30 \mu \mathrm{L}$ of a $0.1 \%$ TFA solution.

\section{Crude Honeybee Venom Extract Characterization: MALDI MS and LC-ESI High Resolution MS (LC-ESI/HRMS)}

The crude venom extract was used to explore its composition through MALDI TOF MS and LC-ESI/HRMS. First, the MALDI target plate was spotted with $1 \mu \mathrm{L}$ of the sample and mixed in the well with either $1 \mu \mathrm{L}$ of 10 $\mathrm{mg} / \mathrm{mL}$ of $\alpha$-cyano-4-hydroxycinnamic acid ( $\alpha \mathrm{CHCA}$ ) dissolved in $70 / 30$ acetonitrile/TFA $0.1 \%$, or $1 \mu \mathrm{L}$ of 10 $\mathrm{mg} / \mathrm{mL}$ of sinapinic acid dissolved in 70/30 acetonitrile/ TFA $0.1 \%$. After complete drying, the samples were submitted to mass spectrometry analysis on a MALDI TOF TOF Ultraflex III (Bruker Daltonics, Germany), which was operated in positive reflectron and positive linear mode for peptide and protein analyses respectively. The peptidic fraction of the venom was acquired in the range $\mathrm{m} / \mathrm{z}$ 100-4000 and calibrated using the Bruker peptide calibrant kit $(m / z$ 1000-3500), whilst the protein fraction was analyzed in the range $6000-50,000 \mathrm{~m} / \mathrm{z}$ using the protein calibrant Bruker kit II $(m / z$ 18,000-66,000).

The remaining $29 \mu \mathrm{L}$ of the venom aliquot was submitted to LC-ESI/HRMS analysis by using an Ulti- 
mate 3000 HPLC system (Dionex, Sunnyvale, CA) coupled to a LTQ-Orbitrap mass spectrometer (Thermo Fisher, San Jose, CA). A C4 Vydac column (150 mm $\times$ $300 \mu \mathrm{m}, 300 \AA$ pore size) was used, operating a linear gradient ramping from $5 \%$ A $(0.1 \%$ aqueous formic acid) to $60 \%$ B (95/5 acetonitrile/ $0.1 \%$ aqueous formic acid) in $30 \mathrm{~min}$. Data were acquired in a range $\mathrm{m} / \mathrm{z}$ $500-2000$ with a nominal resolution of 30,000 (at $\mathrm{m} / \mathrm{z}$ 400 ) in data dependent acquisition, where the first and then the second most intense ions from the MS scan were selected for fragmentation. Data were processed by using the Xcalibur software with the "Xtract" tool for the calculation of deconvoluted average and monoisotopic molecular weight.

\section{Simulation of Honeybee Sting}

In vitro experiments. Four pieces of pig ear and four live honeybees were selected for a single "in vitro" time course experiment. A honeybee was placed adjacent to the porcine skin and held in place using forceps. The defensive instinct induced the honeybee to sting the skin. A time course experiment in which the sting was left in the piece of pig ear for a minimum time of $1 \mathrm{~min}$ to a maximum of $120 \mathrm{~min}(1,30,60,120$, min) was performed. At the end of each time point, the tissues were stored in a $-80^{\circ} \mathrm{C}$ freezer until further analysis. Each time point experiment was repeated three times.

In vivo experiments. Two Wistar rats and two honeybees were used for a single in vivo time course envenomation experiment. Ethical approval was obtained from the Institutional Animal Care and Use Committee, University of Florence, Italy, before starting the experiments. No suffering was inflicted on the rats at each experimental stage. The rats, ranging in weight from 290 to $400 \mathrm{~g}$, were anesthetized with 1 to 2 doses of chloral hydrate $(400 \mathrm{mg} / \mathrm{kg}$ i.p.). When asleep, they had a posterior leg carefully shaved, and they were kept under anesthesia until sacrificed. The honeybees were forced to sting the leg in the same fashion as in the in vitro experiments. A simple time course experiment was outlined so that rats were sacrificed by decapitation at $1 \mathrm{~min}$ and $120 \mathrm{~min}$ after the envenomation event. Each time point experiment was performed in duplicate.

\section{Tissue Staining}

Tissue sections, cut consecutively to those destined for MALDI MSI analysis, were subjected to a fast $\mathrm{H}$ and $\mathrm{E}$ staining protocol. Briefly, sections were fixed in ethanol at decreasing percentages (1 $\mathrm{min}$ in 100\% ethanol, $1 \mathrm{~min}$ in $90 \%$ ethanol, $1 \mathrm{~min}$ in $70 \%$ ethanol). Five seconds washing in water was followed by $1 \mathrm{~min}$ immersion in haematoxylin. The glass slides were then placed in running tap water to remove excess haematoxylin until the water ran clear (about $3 \mathrm{~min}$ ). Subsequently, the tissues were stained for $30 \mathrm{~s}$ in eosin followed by a $5 \mathrm{~s}$ wash in water, $30 \mathrm{~s}$ fixation in $70 \%$ ethanol, $1 \mathrm{~min}$ in $90 \%$ ethanol, and $1 \mathrm{~min}$ in 100\% ethanol. After drying, slides were mounted with coverslips by using DPX.

\section{Mapping HBV Peptides and Proteins by MALDI MSI}

Porcine ear tissue sections (10 $\mu \mathrm{m}$ thick) were cut by using a cryostat kept at $-30^{\circ} \mathrm{C}$ and were then thawmounted on ITO coated conductive glass slides. Sections destined for peptide MALDI MSI were immediately placed in a vacuum dessicator for $30 \mathrm{~min}$. Sections to be subjected to protein MSI were washed in $70 \%$ ethanol for $1 \mathrm{~min}, 90 \%$ ethanol for $30 \mathrm{~s}$, and subsequently immersed for a further $30 \mathrm{~s}$ in a solution of $20 \mathrm{mg} / \mathrm{mL}$ of sinapinic acid in 90/10 ethanol/ $0.1 \%$ TFA and then dried for $15 \mathrm{~min}$ in a vacuum dessicator. Finally, dried sections were sprayed by means of an airbrush (Eclipse HP-CS; Turin, Italy) attached to a $\mathrm{N}_{2}$ line, with either $20 \mathrm{~mL}$ of $20 \mathrm{mg} / \mathrm{mL}$ of $\alpha$-cyano-4-hydroxycinnamic acid ( $\alpha \mathrm{CHCA}$ ) dissolved in 50/50 acetonitrile/TFA $0.1 \%$ (for peptide MALDI MSI), or $25 \mathrm{~mL}$ of $25 \mathrm{mg} / \mathrm{mL}$ of sinapinic acid dissolved in 50/50 acetonitrile/TFA $0.2 \%$ (for protein MALDI MSI). Two passes per spray cycle were used and $30 \mathrm{~s}$ were allowed between cycles. The sections were finally dried in a vacuum dessicator for $10 \mathrm{~min}$ before MALDI MSI analysis. Matrix delocalization was minimized and image reproducibility was observed in serial sections from the same specimen by keeping constant both the $\mathrm{N}_{2}$ pressure and the distance between the sections and the airbrush spray nozzle.

The HBV peptidic fraction was mapped by using an Ultraflex III MALDI TOF/TOF mass spectrometer (Bruker Daltonics, Bremen Germany), in the range $\mathrm{m} / \mathrm{z}$ 100-4000. The instrument was operated in positive reflectron mode and the spatial resolution was set to $300 \mu \mathrm{m}$. The ion sources 1 and 2 were set to 25,000 and $21,500 \mathrm{~V}$, respectively, with the delay time at $60 \mathrm{~ns}$. The HBV protein fraction was investigated in a range between $\mathrm{m} / \mathrm{z} 6000$ and 50,000 in positive linear mode; the delay time was increased to a value of 200 ns. Mass spectra acquisition was performed by using the software Flex Imaging 2.0 and Flex Control 3.0 (Bruker Daltonics) and data processed through Flex Imaging 2.0, Flex Analysis 3.0, and Biomap 3.7.5 (Novartis, Basel, Switzerland) software. Calibration was performed before analysis, depositing either 0.5 $\mu \mathrm{L}$ of crude venom mixed 1:1 with the appropriate matrix and placed $0.5 \mathrm{~cm}$ away and on the left hand side of the area affected by the sting. The $m / z$ values of Api m 4, apamine, MCD, Api m 1, and Api $m 6$ retrieved in the crude venom were used as calibration points for MALDI MSI analysis of HBV peptides and proteins. Normalization through Flex Imaging 2.0 was applied by using a ratio y-mean/y-max of 0.09 . Mass to charge values for image display were se- 
lected with a mass window of $1 \mathrm{Da}$ for peptides and $5 \mathrm{Da}$ for proteins. When Biomap 3.7.5 was used to process the images, ion signals were normalized against the sodium adduct of $\alpha \mathrm{CHCA}(\mathrm{m} / \mathrm{z} 212)$.

\section{Evaluation of $H B V$ Detection Limits in MALDI MSI}

Venom from 30 honeybees was collected and pooled, then dried, weighed, and finally dissolved in $21 \mu \mathrm{L}$ of an aqueous solution of $0.1 \%$ TFA, so that the final concentration of the venom was $102 \mu \mathrm{g} / \mu \mathrm{L}$. Aliquots of $2 \mu \mathrm{L}$ of this solution were prepared to obtain concentrations of $50.1,10.2,1.02$, and $0.102 \mu \mathrm{g} / \mu \mathrm{L}$. A pig ear tissue section was spotted with $0.2 \mu \mathrm{L}$ of each of the above solutions. This effectively resulted in depositing $20.4 \mu \mathrm{g}, 10.0 \mu \mathrm{g}, 2.04 \mu \mathrm{g}, 204 \mathrm{ng}$, and $20.4 \mathrm{ng}$ of venom on the pig skin section. Once dried, the tissue section was spray-coated with $\alpha \mathrm{CHCA}$ matrix for peptide analysis and subjected to MALDI TOF profiling experiments. In this analysis, circular areas of $700 \mu \mathrm{m}$ diameter, corresponding to the diameter of the deposited venom droplet, were selected for laser firing. Five sections (replicates) were submitted to this experiment to compensate for any variability in response due to non-homogeneous matrix deposition and local tissue suppression effects.

\section{Results and Discussion}

Honeybee venom (HBV) is one of the best characterized venoms amongst hymenoptera. Its composition, isolation, characterization, and the cloning of its bioactive species induced physiological damages and use in therapeutic treatments, are some of the aspects so far investigated [9, 22, 38, 39].

Here, the use of a novel technology named MALDI MSI is applied to the detection of HBV in envenomed tissues. A time course MALDI MSI experiment was designed and performed to establish whether the venom components were detectable and their limits of detection as well as their diffusion behavior within the tissue over time. In particular, two toxins (apamine and MCD) and three allergens (Api m 4, Api m 6, and Api $\mathrm{m} 1$ ) were investigated. Molecular mass images of venom distribution were obtained for all of these biomolecules, showing the potential of this technology for further and more in-depth studies of venom action and the organism's response.

A preliminary structural characterization was performed to find the most readily detected HBV molecular species by using MALDI TOF MS and LC-ESI/ HRMS. In particular, optimal instrumental conditions were determined to enhance ionization of the interested biomolecules in MALDI MSI.

\section{$H B V$ Characterization}

Peptide range. Among honeybee venom peptides, the most popular and characterized neurotoxins are apamine and MCD. Api $\mathrm{m} \mathrm{4}$, although being a peptide, is actually considered a minor allergen. A simple characterization of the crude venom extract, in particular of its peptidic fraction, by MALDI TOF MS allowed the detection of these three species at $\mathrm{m} / \mathrm{z}$ of 2026.82, 2586.53 and 2845.63, respectively, in agreement with the theoretical $\mathrm{m} / \mathrm{z}$ value; apamine has two disulphide bridges and an amidated C-terminal histidine residue [40], thus protonated apamine has a theoretical monoisotopic $\mathrm{m} / \mathrm{z}$ of 2026.86. MCD also has two disulphide bridges and an amidation at the C-terminal asparagine residue [27], thus exhibiting a theoretical monoisotopic $\mathrm{m} / \mathrm{z}$ of 2586.38. Processed Api $m 4$ exhibits an amidation at the C-terminal glutamine residue and has a theoretical monoisotopic $\mathrm{m} / \mathrm{z}$ of 2845.74 [6].

Protein range. The MALDI MS analysis of the HBV protein fraction was performed using either $\alpha \mathrm{CHCA}$ or sinapinic acid as matrices, obtaining complementary information. An ion signal at $m / z 7589.3$ was found that could correspond to the MW of the protein Api m 6, in particular to the isoform Api m 6.03 (average MW $7588.1 \mathrm{Da}$ ). No other isoforms of this allergen were detected by conventional MALDI MS analysis. Api $m 1$ was also detected in the MALDI MS analysis of the crude venom extract. An ion signal at $m / z 15,240.2$ was in fact observed $(15,239.2 \mathrm{Da}$ is the theoretical average MW through cysteine linked disulphide bridges). This ion signal was accompanied by a rich population of other peaks distributed around $16.3 \mathrm{kDa}$ and possibly related to micro heterogeneous Api m 1 glycosylation. Scheme 1 shows the possible glycostructures observed, which agree with those reported by Shipolini and collaborators [15] and Hollander et al. [41]. Higher $\mathrm{m} / \mathrm{z}$ protein signals were also detected (up to $50 \mathrm{kDa}$ ) but the intensity and the resolution were too poor to proceed with any $\mathrm{m} / \mathrm{z}$ assignment.

The LC-ESI/HRMS analysis on the crude HBV extract gave the accurate MW of the intact HBV protein species, thus supporting their detection via MALDI TOF MS analysis and enabling the detection of other isoforms. In particular for this analysis, Scheme 1 reports the allergen Api m 6 detected at 6913.4 Da as 8-71 (theoretical monoisotopic MW 6913.4 Da), at 7174.5 Da as 5-71 (Api m 6.01, theoretical monoisotopic MW $7174.5 \mathrm{Da}$ ), and at $7384.7 \mathrm{Da}$ as 5-73 (Api m 6.02, theoretical monoisotopic MW 7384.7 Da). In the LCESI/HRMS experiments, Api $\mathrm{m} 1$ was detected as a secreted protein with no glycosylation at an average MW of $15,239.0 \mathrm{Da}$ and at a monoisotopic MW of $15,229.017 \mathrm{Da}(15,229.022 \mathrm{Da}$ is the theoretical monoisotopic MW with cysteine linked disulphide bridges). The presence of at least four potential Api m 1 glycoisoforms was also observed at a monoisotopic MW of $15,797.2,15,959.3,16,122.4,16,284.4$, and 16,674.4 Da as 


\begin{tabular}{|c|c|c|c|c|}
\hline Allergen Api $m 6$ & $\begin{array}{l}\text { MALDI TOF } \\
\text { MS (average } \\
\text { m/z) }\end{array}$ & $\begin{array}{c}\text { LC HR ESI } \\
\text { MS (average } \\
\text { MW) }\end{array}$ & $\begin{array}{c}\text { MALDI MSI } \\
\text { (average } \\
\text { m/z) }\end{array}$ & Sequence \\
\hline Apim 6 & - & 6918.4 & 6919.7 & 8.71 \\
\hline Apim 6.03 & - & 7178.5 & 7179.5 & 5.71 \\
\hline Apim 6 & - & - & 7384.8 & $\frac{3.71}{37}$ \\
\hline Apim 6 & - & $\cdot$ & 7441.8 & $2 \cdot 71$ \\
\hline Apim 6.02 & $-\dot{0}$ & 7388.7 & & 5.73 \\
\hline Apim 6.01 & 7589.3 & $\cdot$ & 7589.1 & 1.71 \\
\hline Apim 6.04 & & $\cdot$ & 7798.4 & 1.73 \\
\hline Api m 1 & & & & Glyco-structure \\
\hline Allergen Api m 1 & 15240.3 & 15239.0 & 15240.1 & \\
\hline glyco Api m 1_1 & 15808.2 & 15807.3 & - & $\theta=-0$ \\
\hline glyco Api m 1_2 & 15971.3 & 15969.5 & 15971.1 & \\
\hline glyco Api m1_3 & - & - & 16117.7 & \\
\hline glyco Api m 1_4 & 16132.2 & 16131.3 & - & \\
\hline glyco Api m 1_5 & 16294.2 & 16293.8 & - & \\
\hline glyco Api m 1_6 & 16337.6 & - & - & \\
\hline glyco Api $m$ 1_7 & 16483.7 & - & - & \\
\hline glyco Api i 1 _8 & 16684.4 & 16683.2 & - & \\
\hline glyco Api m 1_9 & - & - & 16846.8 & \\
\hline
\end{tabular}

Scheme 1. Api m 6 and Api $\mathrm{m} 1$ detection by mass spectrometry. The MW and $m / z$ values detected for Api $\mathrm{m} 1$ and Api $\mathrm{m} 6$ along with their isoforms are reported. The venom was extracted and analyzed by MALDI TOF MS conventional analysis (column 2) and LC-ESI/HRMS analysis (column 3). Data deconvolution, from the latter, generated average and monoisotopic MW, but only the average mass values are reported here. MALDI MSI experiments performed on the envenomed tissue allowed the detection of both Api $\mathrm{m} 1$ and Api m 6 (column 4). Primary sequence and the glycosylated structure linked to the protein are reported for Api $\mathrm{m}$ 6 and Api m 1 isoforms, respectively. Monosaccharide residues are depicted as follows: fucose residue as a triangle, mannose/ galactose residue as a circle, $\mathrm{N}$-acetylglucosamine residue as a square.

shown in Figure S1 of Supplemental Material, which can be found in the electronic version of this article; $(15,807.3,15,969.5,16,131.3,16,293.8$, and 16,683.2 Da are the average MW respectively, reported in Scheme 1). Thus, it was possible to confirm the presence of three of the glyco-Api $\mathrm{m} 1$ ion signals previously detected by MALDI TOF MS, once again enhancing confidence in the correct MALDI $\mathrm{m} / \mathrm{z}$ attribution; this is, in turn, also valuable both for calibration of the MALDI mass spectrometer instrument operating in MSI mode and for post analysis recalibration.

The presence of the two neurotoxin peptides and three allergens was further confirmed by a bottom up approach on the venom tryptic digest, where the resulting peptidic mixture was submitted to shotgun proteomic approach by MALDI TOF MS and MS/MS analysis and LC-ESI HRMS/MS analysis.

In summary, the HBV has been characterized by conventional MALDI MS analysis, by peptide mass fingerprinting and MS/MS analysis, and finally by LC-ESI/HRMS for accurate MW determination. Figure S1A, B, and C, (Supplemental Material) show an overview of the global characterization strategy followed in this study, including examples of analysis for Api m 4,

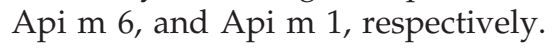

\section{Evaluating Venom Detection Limits in MALDI MSI}

MALDI MSI is undoubtedly very informative from a qualitative point of view; nonetheless the feasibility of quantitative analysis is very much debated (as expected dealing with MALDI MS) and it is regarded as a weakness of this technique. Whether a signal is not detected because the species is not present, or is beyond the detection limit, appears to be, for MALDI MSI in particular, a dilemma. Indeed, this technology may exhibit lower sensitivity in comparison to conventional MALDI MS application; this is because ion suppression (often selective) might be caused not only by the matrix but also by the presence of salts and the tissue itself. In the present study, the authors evaluated MALDI MSI detection limits by monitoring HBV components in envenomed tissues.

HBV was spotted at decreasing concentrations on a $10 \mu \mathrm{m}$ tissue section from pig ear sample, which was then spray coated with $\alpha$ CHCA to simulate its deposition in conventional imaging analysis. Five replicates were analyzed and processed; factors including uneven matrix distribution, tissue specific suppression, and suppression due to competitive ionization were then taken into account and normalized. HBV was deposited only in the region between the epidermis and the cartilage (where the venom is mostly detected); results obtained relate exclusively to this area and are not applicable, without further investigation, to other tissue regions or to different tissue types. Even within the same tissue, in fact, structurally different regions may have different ion suppression effects, thus influencing ionization yields. Only the HBV peptidic components (apamine, MCD, and Api m 4) were considered for this analysis. This choice was primarily made by considering the characteristics of Api m 4. It in fact has a molecular weight of only $2.8 \mathrm{kDa}$, it exhibits a very good ionization yield and it represents $45 \%$ to $50 \%$ of the venom dry weight. It is then reasonable to hypothesize that, for concentrations at which it is not detectable, it would be unlikely that higher molecular weight proteins, expressed in less than $10 \%$ of the venom dry weight, would be detected. On the basis of the amount of venom deposited and the percentage of Api m 4 ( $45 \%$ to $50 \%)$, apamine (1\% to $2 \%)$, and MCD (1\% to $2 \%)$ contained in it [12], it was possible to estimate the amount of these peptides deposited on the tissue (Table 1). Api $\mathrm{m} 4$ exhibits the maximum ion intensity when spotting $2.04 \mu \mathrm{g}$ of venom (1/10 dilution of the original stock solution, $10.2 \mu \mathrm{g} / \mu \mathrm{L})$; here the absolute amount of Api m 4 was calculated to be $0.92-1.02 \mu \mathrm{g}$; Figure S2 (Supplemental Material) shows a set of Api m 4 MALDI MS spectra for one replicate as an example. The same trend is observed for MCD (absolute amount corre- 
Table 1. Amount of HBV spotted on tissue

\begin{tabular}{lccrr}
\hline & Amount of & & Apamine & MCD abs. \\
venom deposited & Api m 4 abs. amount & abs. amount & $0.20-0.40 \mu \mathrm{g}$ \\
\hline \hline 102 & $20.4 \mu \mathrm{g}$ & $9.2-10.2 \mu \mathrm{g}$ & $0.20-0.40 \mu \mathrm{g}$ & $0.10-0.20 \mu \mathrm{g}$ \\
50.1 & $10.2 \mu \mathrm{g}$ & $4.6-5.1 \mu \mathrm{g}$ & $0.10-0.20 \mu \mathrm{g}$ & $20.4-40.8 \mathrm{ng}$ \\
10.2 & $2.04 \mu \mathrm{g}$ & $0.92-1.02 \mu \mathrm{g}$ & $20.4-40.8 \mathrm{ng}$ & $0.92-1.84 \mathrm{ng}$ \\
1.02 & $204 \mathrm{ng}$ & $91.8-102 \mathrm{ng}$ & $0.92-1.84 \mathrm{ng}$ & $0.2-0.40 \mathrm{ng}$ \\
0.102 & $20.4 \mathrm{ng}$ & $9.2-10.2 \mathrm{ng}$ & $0.2-0.40 \mathrm{ng}$ & \\
\hline
\end{tabular}

sponding to maximum intensity is 20.4-40.8 $\mathrm{ng}$ ). Apamine maximum intensity is variable in the replicates: it is observed when spotting both 2.04 and $10.0 \mu \mathrm{g}$ of venom corresponding to $1 / 2$ and $1 / 10$ dilution of the original stock solution (absolute amount deposited, 20.4-40.8 ng and $0.10-0.20 \mu \mathrm{g}$, respectively); nonetheless the highest intensity is observed at the $1 / 2$ dilution; generally, the maximum HBV concentration used did not produce the most intense signal. This is in agreement with the very nature of MALDI ionization, where the matrix/analyte ratio is crucial for maximum ionization yields. As for the detection limits, data show that with the lowest HBV amount spotted in MALDI Imaging experiments, Api m 4 could still be easily detected $(9.2-10.2 \mathrm{ng}, \sim 6.8 \mathrm{pmol})$ in the five replicates, whereas for apamine and MCD the approximate detection limit is $0.2-0.4 \mathrm{ng}$ on tissue. Figure S3 (Supplemental Material) summarizes results for the three peptides in two graphs, where the average ion intensity for each species is plotted versus the aliquot concentration from which 1 $\mu \mathrm{L}$ of venom was withdrawn and spotted. Considering the amount of HBV injected per sting reported by Freeman and coworkers [31] (50 $\mu \mathrm{g})$, the minimum amount of Apamine and MCD detected is $\sim 3$ orders of magnitude lower than the actual amount contained in the venom (considering that they are present at 1\%); the minimum amount of Api $\mathrm{m} 4$ detected in the analysis, corresponds to 4 orders of magnitude lower than the actual amount contained in the volume of the venom delivered by a single sting (considering Api $\mathrm{m} 4$ is present as $\sim 45 \%$ ). Conclusively, considering the amounts of venom spotted in this experiment, the MALDI MSI detection limits were $\leq 9.7 \mathrm{ng}$ for Api $\mathrm{m} 4$ and $\leq 0.2-0.3 \mathrm{ng}$ for apamine and MCD; this analysis was considered satisfactory for the purpose and the detection limits suitable for HBV MALDI MSI analysis.

\section{MALDI MSI Analysis of Envenomed Tissues}

In vitro analysis using honeybees and porcine ears. The MALDI MSI analysis of the HBV peptidic fraction proved to be successful in mapping HBV within a biological tissue following a honeybee sting at different time intervals. The shortest time point of $1 \mathrm{~min}$ was chosen because it had previously been reported that in both rabbit skin and various artificial media, the sting embedded itself progressively deeper over a period of $\sim 30 \mathrm{~s}$; at the end of this period, at least two-thirds of its length was embedded [4] and venom inoculation is thought to start at this time.

It is important to bear in mind that this is not a time course experiment in real terms. Different pig ear samples were used, which were then stung and left to incubate for different time periods. The tissue morphology, compactness, and thickness of the different skin layers were observed to vary by histological inspection so that a precise estimation of venom diffusion at different times by simple comparison is not correct; nonetheless MS images of apamine, MCD, and Api $\mathrm{m} 4$ were obtained clearly showing their localization within the tissue. Figure 1a shows, as an example, the $\mathrm{H}$ and $\mathrm{E}$ histological image of a $10 \mu \mathrm{m}$ section cut from a porcine skin sample stung by a honeybee (as a preliminary experiment, $5 \mathrm{~min}$ were allowed to pass before freezing and slicing the ear tissue). This section was the serial section to the one submitted to MALDI MSI. Five min after the sting, the presence of an irritated area was

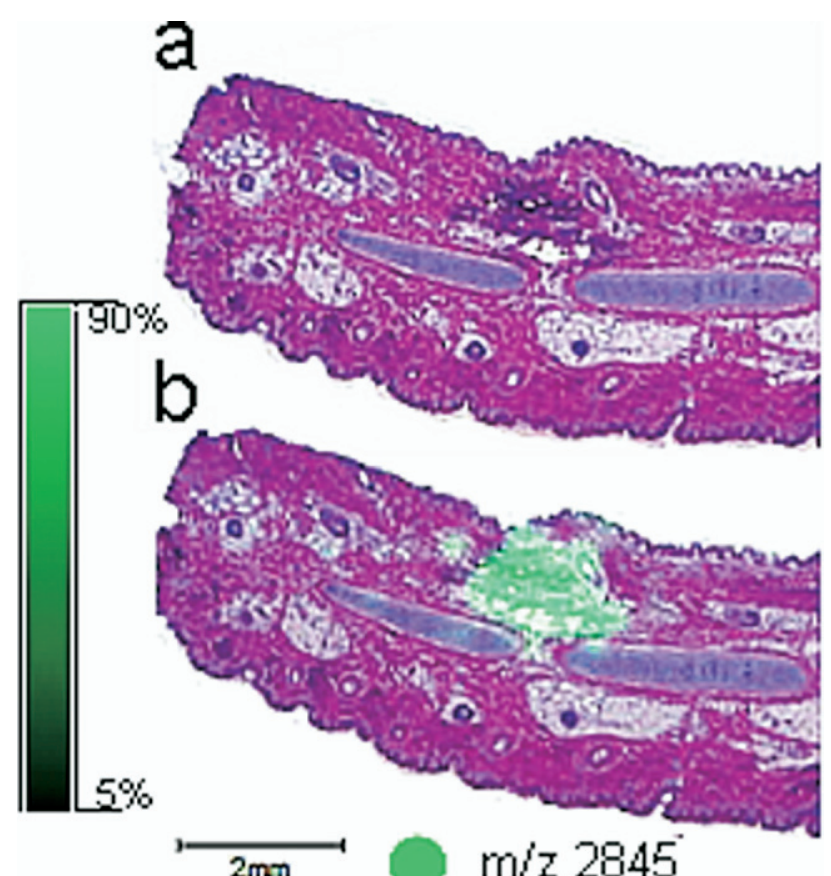

Figure 1. Tissue optical and MALDI MS images at $5 \mathrm{~min}$ post-envenomation. A and E histological image of a $10 \mu \mathrm{m}$ pig ear section shows an irritated (darker) area (a); by using the Flex Imaging 3.0 software, MALDI MS image of Api m $4(\mathrm{~m} / \mathrm{z} 2845)$ was superimposed showing that the irritated area is, in fact, characterized by the presence of this allergen (b). 
already observed. This area was presumably affected by the presence of the diffused venom and this hypothesis was confirmed by the superimposition of the Api m 4 MS image on the histological one (Figure 1b). As anticipated, the presence of the venom in the tissue was observed throughout the time course MALDI MSI experiment (analysis at 1, 30, 60, and $120 \mathrm{~min}$ postenvenomation, Figure 2). Apamine, MCD and Api $m 4$ MS images are shown in Figure 2 as superimposed with the MS image of phosphatidylcholine $(\mathrm{m} / \mathrm{z}$ 184). This facilitates visualization of the section and the relative spatial distribution of the peptides. Although neither venom quantitation nor a precise estimation of the tissue total area infiltrated by the venom over time was at this stage feasible, it was possible to observe a general trend showing a progressive decrease in the detection of the HBV peptides over time.
Bioactive HBV peptides are readily mapped at $1 \mathrm{~min}$ post-envenomation. In particular, Api $\mathrm{m} 4$ is detected at 1 min post-envenomation, it is then observed to spread after $30 \mathrm{~min}$, and seems to accumulate in the proximity of the cartilage $60 \mathrm{~min}$ after the envenomation event; this accumulation might be due to the cartilage possibly acting, somehow, as a barrier to the longitudinal diffusion. Figure S4 (Supplemental Material) shows the superimposition of the MS images with the histological images of the tissue section thus supporting this last observation.

MCD and apamine were also detected in the MALDI MSI experiment and had the same ratios of intensity peak intensity proportions, as that observed in conventional MALDI MS analysis of the extracted venom. This suggests that if the tissue is causing ion suppression, this event is not selective toward any specific peptide in
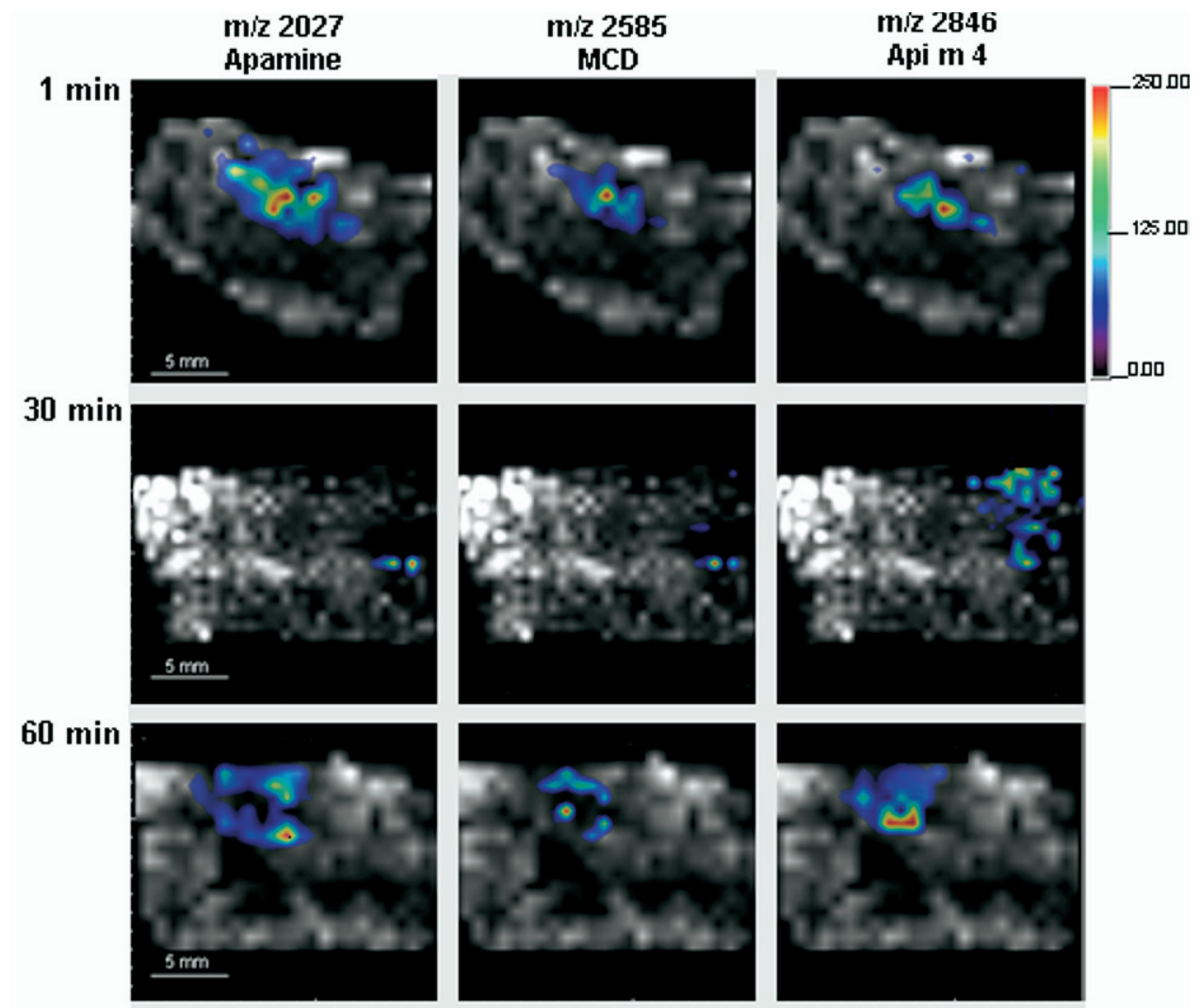

$120 \mathrm{~min}$
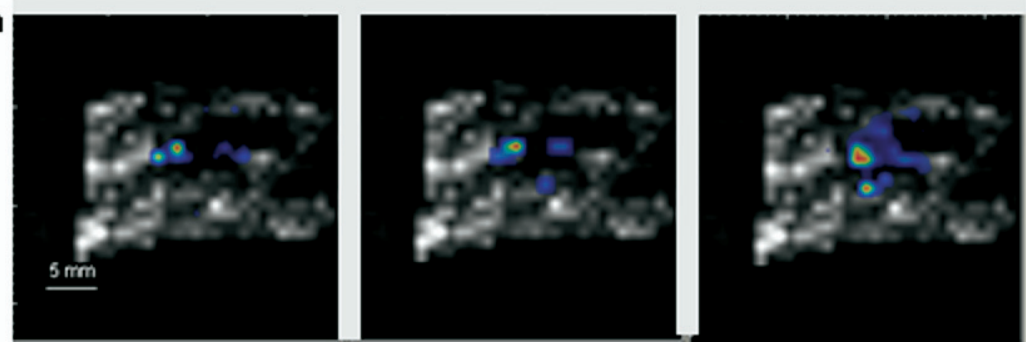

Figure 2. MALDI MS images of HBV peptides. By using the Biomap 3.7 .5 software, distribution maps of Apamine, MCD, and Api $\mathrm{m} 4$ at 1, 30, 60, and 120 min post-envenomation were obtained. The MS images were normalized against the $\alpha$ CHCA sodium adduct $(m / z 212)$ distribution. The figure shows the peptide MS images (in color) superimposed with the MS image of phosphatidylcholine $(\mathrm{m} / \mathrm{z}$ 184, gray scale) to visualize the tissue section and the spatial distribution of Apamine, MCD and Api m 4 within it. 
particular. MCD distribution maps over time show detection at $1 \mathrm{~min}$, a marked longitudinal diffusion at 30 min, with high density at the bottom of the image belonging to MCD longitudinal diffusion; once again, the maximum area affected is retrieved at $60 \mathrm{~min}$ post-envenomation. Finally, Apamine is readily detected after $1 \mathrm{~min}$ post-envenomation and seems to undergo accumulation at $60 \mathrm{~min}$ as with Api m 4; the apamine signal exhibits the same general trend as that for $\mathrm{MCD}$, the only difference being that the ion signal is generally more intense. At 120 min post-envenomation, localization maps for the three peptides are considerably reduced, although Api m 4 and MCD are largely detected also within the cartilage; apamine might not be as well-detected in that area if the cartilage exerts a selective ion suppression towards this ion. Since the tissue was not viable at the time of the experiment, the hypothesis that peptides were degraded by the tissue was, in the first instance, ruled out; there could be other reasons for the observed reduction in signal detection. Over time, HBV peptides progressively diffuse and, therefore, they might reach a concentration below the detection limits. The increased diffusion could also lead to a ratio analyte/matrix value, which falls outside the minimum for detection.
MALDI MSI analysis on the protein fraction of HBV also showed very interesting results, including insights into the structure of the allergens studied (Api $\mathrm{m} 6$ and Api m 1). MALDI MSI time course analysis on the HBV protein fraction allowed mapping of the Api $m$ 6 (mature protein $\mathrm{MW} 7588.1 \mathrm{Da}$ ) and Api $\mathrm{m} 1$ (mature protein MW 15,239.2 Da) distribution, and confirming a few features of their heterogeneous nature, due to misassembly and the presence of glycoisoforms, respectively.

In particular, MALDI MSI analysis revealed a more heterogeneous composition for Api $\mathrm{m} 6$ than that reported. It is known, in fact, that Api m 6 comprises 4 isoforms: 1-71 (Api 6.01), 1-73 (Api m 6.04), 5-71 (Api m 6.03), and 5-73 (Api m 6.02), which are all allergenic [20]. Upon MALDI MSI of the $30 \mathrm{~min}$ post-envenomation tissue, co-localized molecular images of Api m 6.01, Api m 6.0, and Api 6.04 were obtained (Figure 3). Figure 3 reports Api $\mathrm{m} 6$ MS images superimposed with the MS image of a protein at $m / z 11,233$, which is distributed throughout the section except in the cartilage. This aids in visualization of the section, the cartilage (negative image), and the relative spatial distribution of Api m 6. By retrieving the MS spectrum associated with the highest pixel intensity of isoform Api m 6.03,

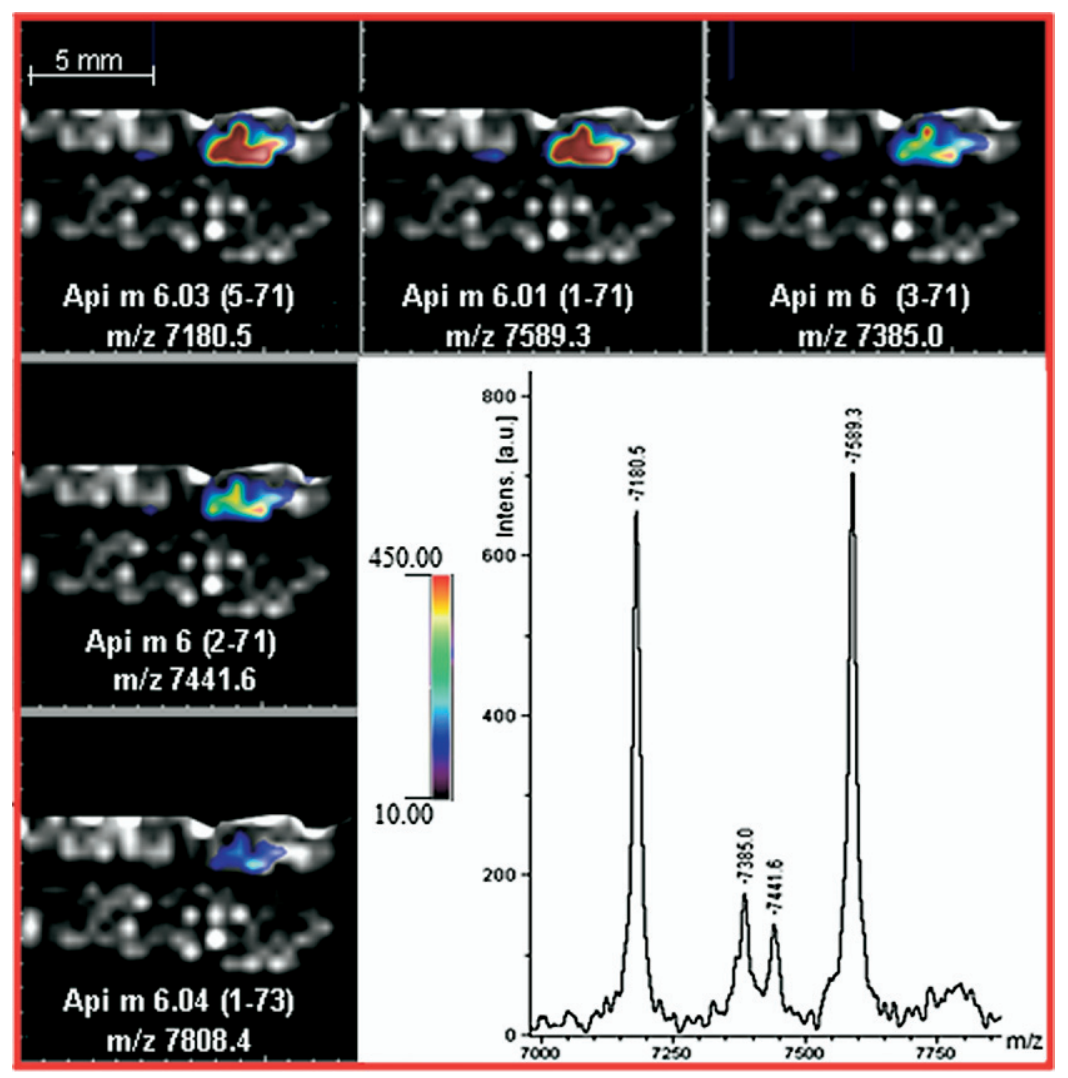

Figure 3. MALDI MS images of allergen Api $\mathrm{m}$ 6. Detection and distribution maps of Api $\mathrm{m} 6$ isoforms at $30 \mathrm{~min}$ post-envenomation along with the corresponding MALDI MS spectrum are illustrated. Api m 6 MALDI images (in color) were superimposed with the MALDI image of a protein at $m / z 11,233$ (grey scale), which localizes across the whole tissue section, except in the cartilage. This allows visualization of the section, of the cartilage (negative image), and a better evaluation of Api $\mathrm{m}$ 6 relative spatial distribution. 
it was observed that, besides those isoforms, the spectrum exhibited two other interesting signals at $\mathrm{m} / \mathrm{z} 7741.6$ and 7385.0 related to Api $\mathrm{m} 6$ sequence: 2-71 and 3-71 (Figure 3). A very low intensity peak $(\mathrm{S} / \mathrm{N} 1.7)$ at $\mathrm{m} / \mathrm{z} 6919.3$ also revealed the presence of isoform 8-71 (Figure S5, Supplemental Material). It is important to bear in mind that Api $\mathrm{m} 6$ is not reported to be subjected to any modification upon venom inoculation and interaction with the tissue [20]; this is confirmed by the observation that most of the above species were present in the crude venom extract (Scheme 1).

This multi-isoform detection adds a further level of complexity to the expression of this allergen, which seems to exhibit a much higher degree of misassembly, and it would be interesting to investigate whether or not these newly discovered forms are also allergenic. Another interesting observation can be made by examining the MS images reported in Figure 3; in contrast to the work of Kettner et al. [20], which suggested that Api $\mathrm{m} 6$ isoforms are present in approximately equimolar amounts, the MALDI MSI analysis found that Api m 6.02 was not detected, whilst Api m 6.04 exhibited a remarkably lower density map compared with Api m 6.03 and Api m 6.01, which are the only two isoforms with comparable ion density distributions. Although the MALDI ionization yield is not proportional to concentration, since those three isoforms co-localize and are not significantly structurally different, it would be hard to invoke a differential and substantial ionization yield or a specific tissue suppression to justify such a remarkable difference in the density maps; it is therefore hypothesized that these isoforms may, in fact, not be present in equimolar amounts.

Exploring the area where Api $\mathrm{m} 6$ was distributed, co-localization of Api $\mathrm{m} 1$ signal at $\mathrm{m} / \mathrm{z}$ 15,240 was observed, as shown in the corresponding MS spectrum extracted from the MALDI MSI run (Figure S5). Three other ion signals at $m / z 15,971,16,116$, and 16,846 were detected in that area and were compatible with three Api $\mathrm{m} 1$ isoforms exhibiting different glycation levels (Scheme 1).

The MALDI MSI time course on the HBV protein fraction produced images of Api m 1 and Api m 6 at 30 and $60 \mathrm{~min}$ post-envenomation (Figure 4). In Figure 4, Api $\mathrm{m} 1$ and Api m 6 MS images are shown superimposed with the digital scan of the tissue section, thus allowing a better evaluation of Api $\mathrm{m} 6$ relative spatial distribution. At $30 \mathrm{~min}$ post-envenomation, Api m 6 and Api m 1 exhibited a similar diffusion pattern, which is also very different from the one observed for HBV peptides, appearing much less diffuse.

The MALDI MSI time course experiment consistently failed to produce distribution maps for Api $\mathrm{m} 6$ and Api $\mathrm{m} 1$ allergens, both at $1 \mathrm{~min}$ and $120 \mathrm{~min}$ post-envenomation. One possible hypothesis to test further could simply be that at $1 \mathrm{~min}$ the venom has not been fully inoculated and spread sufficiently for the Api $\mathrm{m} 1$ and Api $\mathrm{m} 6$ ion signals to fall within the detection limits of the instrument; at $120 \mathrm{~min}$ the opposite situation (venom spreading and greater diffusion) may cause the same result where allergen concentration is insufficient to be detected. Indeed, already at $60 \mathrm{~min}$ post-envenomation, the areas in which Api m 6 and Api m 1 were observed are considerably reduced compared with those observed at $30 \mathrm{~min}$ post-venom inoculation.

In vivo experiments using honeybees and rats. An in vivo analysis of envenomed tissues was also attempted to prove the potential of MALDI MSI to simultaneously map venom action and organism response. Initial experiments were performed using anaesthetized rats instead of pig ears in a similar fashion to previously described in vitro experiments. Two time points were assessed analyzing tissues after 1 and $120 \mathrm{~min}$ from the sting event. Results were encouraging but also high-

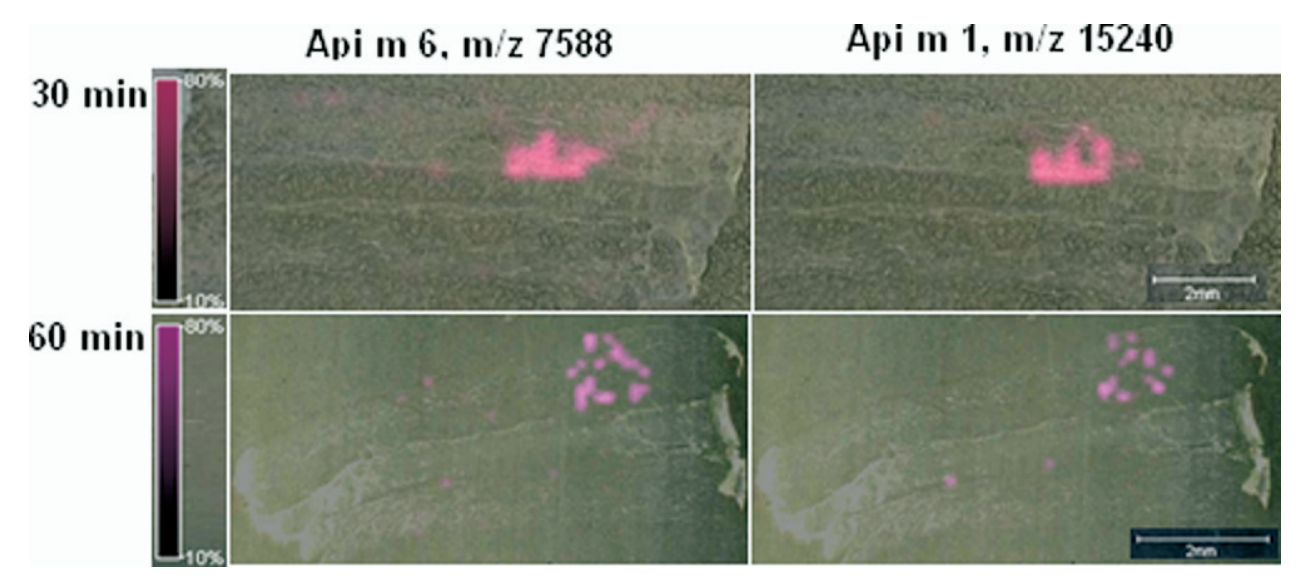

Figure 4. MALDI MS images of allergens Api m 6 and Api m 1. Distribution maps at 30 and 60 min post-envenomation are shown. Api $\mathrm{m} 6$ and Api m 1 MALDI images were superimposed with the digital scan of the tissue section, thus allowing a better evaluation of the allergen's relative spatial distribution. 


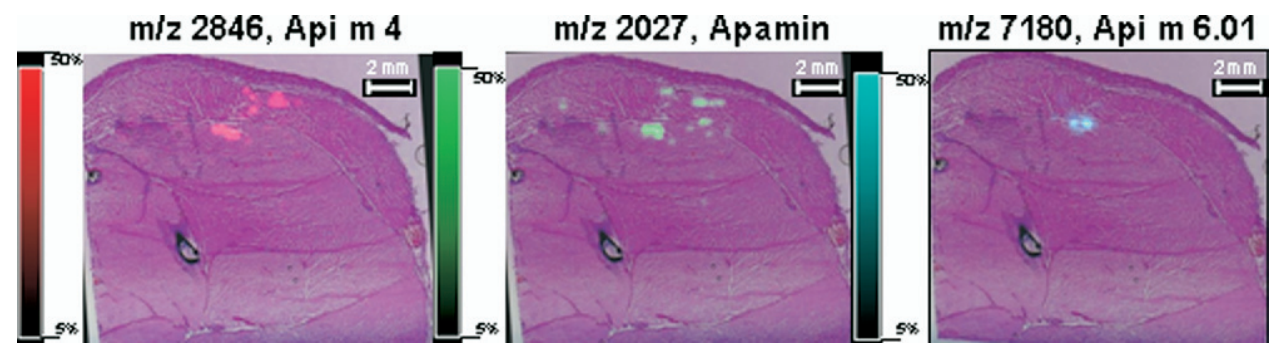

Figure 5. MALDI MS images of Apamine, Api m 4, and Api $\mathrm{m} 6$ from the in vivo time course experiment. Distribution maps within the envenomed tissue at $1 \mathrm{~min}$ post-envenomation are shown superimposed with the correspondent $\mathrm{H}$ and $\mathrm{E}$ histological images.

lighted areas for improvement; data showed that the in vivo MALDI MSI analysis of envenomed tissues generally yields HBV ion signals of lower intensity. The ion images showed a rational localization within the tissue but the venom was observed in smaller areas compared with those observed in the pig ear samples at the same time points. Api $\mathrm{m} \mathrm{4}$, apamine, and Api $\mathrm{m} 6$ were detected at $1 \mathrm{~min}$, although the diffusion in rat muscle seemed to be more contained around the sting, compared with that observed in the porcine sample (Figure 5); at $120 \mathrm{~min}$ post-envenomation detection is extremely poor. Apamine and Api $\mathrm{m} 4$ were detected in only six positions whilst Api $\mathrm{m} 6$ was no longer observed, whereas Api $\mathrm{m} 1$ was detected (data not shown). For results at $1 \mathrm{~min}$ and $120 \mathrm{~min}$, it can be hypothesized that in these cases, besides diffusion, the tissue components may play a role; being biologically active at the time of the sting event, it could react to the venom leading to degradation. To confirm whether it is diffusion or degradation that leads to the observed effects requires further investigation.

In an attempt to detect a tissue response to HBV, a more careful exploration of the mass range between 10-25 kDa (protein $\mathrm{m} / \mathrm{z}$ range) and 300-900 Da (lipid range) was carried out. MALDI MSI and MALDI MS data processing revealed the presence of the sulphidoleukotrienes LTC4 and LTD4 at $\mathrm{m} / \mathrm{z} 497.2$ and 624.3, respectively (theoretical MW 496.3 and $623.3 \mathrm{Da}$ ), as shown in Figure 6 (upper panel). Sulphidoleukotrienes are important markers of an inflammatory event such as the one caused by HBV sting. At 1 min postenvenomation they were found nicely co-distributed with the sting, which was left within the tissue, and in close proximity. At $1 \mathrm{~min}$ post-envenomation, an ion signal at $m / z$ 13,760, (Figure 6 upper panel) compatible with the molecular weight of interleukin 4 (IL-4, theoretical $\mathrm{MW}$ of mature protein with one disulphide bridge is 13,761 Da) was also detected (Figure 6, upper panel). Although, the presence of IL-4 must be further investigated and confirmed, this result would be in partial agreement with the work of Mustafa and coworkers [11], who reported the presence of sulphidoleukotrienes within 5 min of a sting, whilst IL-4 was induced at later time points. At $120 \mathrm{~min}$ postenvenomation, the distribution maps for LCT4, LTD4, and the putative IL-4 change (Figure 6, lower panel). LTD4 appears much more widespread, whilst LTC4 is almost undetectable, whereas IL-4 is localized in an area close to the sting.

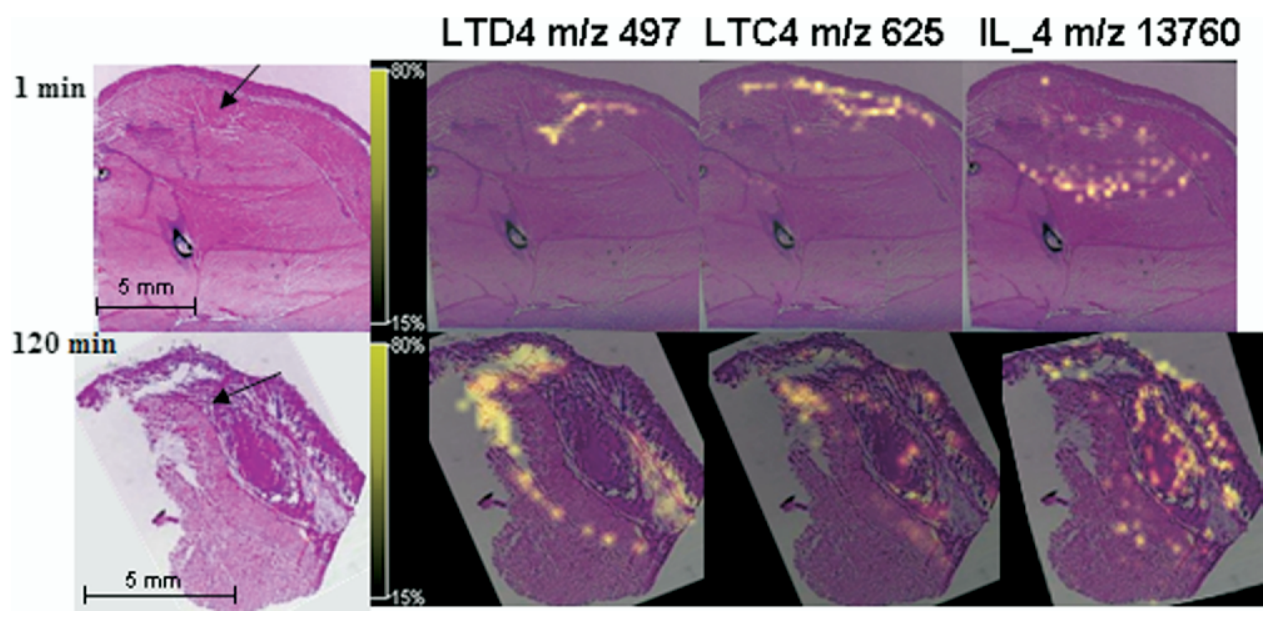

Figure 6. MALDI MS images of the tissue biochemical response to envenomation. Figure reports the $\mathrm{H}$ and $\mathrm{H}$ histological image of the rat leg muscle section (the arrows indicate the original position of the sting) along with the MS images of two sulphidoleukotrienes (LTC4 and LTD4) and a putative cytokine (IL-4) at 1 and 120 min post-envenomation. 


\section{Conclusions}

The work described in this paper demonstrates the ability of MALDI MSI to provide molecular maps of HBV in envenomed tissues over time. This technology offers the opportunity of monitoring, simultaneously, HBV presence and diffusion over time, as well as detecting the host organism response. This raises the possibility that MALDI MSI could be used in the preclinical trial stage development of more efficient venom immunotherapies, which in the future could be tailored to individuals.

\section{Acknowledgments}

The authors gratefully acknowledge Ente Cassa di Risparmio Firenze for salary support (S.F.). The authors thank Dr. Pietro Traldi for fruitful discussions. Professors M.R. Clench and N. Woodroofe are gratefully acknowledged for the valuable comments and suggestions during the first and the second revision of the manuscript.

\section{References}

1. Banks, B. E. C.; Shipolini, R. A. Chemistry and Pharmacology of Honeybee Venom. In Venoms of the Hymenoptera, Piek, T., Ed.; Academic Press: London, 1986; p. 329-416.

2. Habermann, E. Chemistry, Pharmacology, and Toxicology of Bee, Wasp, and Hornet Venoms. In Venomous Animals and Their Venoms, Bucherl, W., Buckley, E. E., Eds.; Academic Press: New York, 1971; p. 61-93.

3. Vetter, R. S.; Visscher, P. K.; Camazine, S. Mass Envenomations by Honey Bees and Wasps. West. I. Med. 1999, 170, 223-227.

4. Schumacher, M. J.; Tveten, M. S.; Egen, N. B. Rate and Quantity of Delivery of Venom from Honeybee Stings. J. Allergy Clin. Immunol. 1994, 93, 831-835.

5. Kreil, G. Biosynthesis of Melittin, a Toxic Peptide from Bee Venom. Eur. J. Biochem. 1973, 33, 558-566.

6. Kreil, G. Hyaluronidases: A group of neglected enzymes. Prot. Sci. 1995, 4, 1666-1669.

7. Hoffman, D. R. Hymenoptera Venom Proteins. Nat. Toxins 1996, 2, 169-186.

8. Grunwald, T.; Bockisch, B.; Spillner, E.; Ring, J. Bredehorst, R., Ollert, M. W. Molecular Cloning and Expression in Insect Cells of Honeybee Venom Allergen Acid Phosphatase (Api m 3). J. Allergy Clin. Immunol. 2006, 117, 848-854.

9. Peiren, N.; Vanrobaeys, F.; de Graaf, D. C.; Devreese, B.; Van Beeumen J.; Jacobs F. J. The protein composition of honeybee venom reconsidered by a proteomic approach. Biochim. Biophys. Acta 2005, 1752, 1-5.

10. Peiren. N.; de Graaf, D. C.; Brunain, M.; Bridts, C. H.; Ebo, D. G.; Stevens, W. J.; Jacobs, F. J. Molecular Cloning and Expression of Icarapin, a Novel IgE-Binding Bee Venom Protein. FEBS Lett. 2006, 580, 4895-4899

11. Mustafa, F. B.; Ng, F. S. P.; Nguyen, T. H.; Lim, L. H. K. Honeybee Venom Secretory Phospholipase A2 Induces Leukotriene Production But Not Histamine Release from Human Basophils. Clin. Exp. Immunol. 2007, 151, 94-100.

12. de Lima, P. R.; Brochetto-Braga, M. R. Hymenoptera Venom Review Focusing on Apis mellifera. J. Venom Anim. Toxins Trop. Dis. 2003, 9, $149-162$.

13. David, B. Current Knowledge on Molecular Structure of Allergens. Rev. Fr. Allergol. 1999 [Numero special], 2-5.

14. de Weck, A. L.; Sanza, M. L. Cellular Allergen Stimulation Test (CAST), a Review. J. Invest. Allergol. Clin. Immunolol. 2004, 14, 253-273.
15. Shipolini, R. A.; Callewaert, G. L.; Cottrell, R. C.; Vernon, C. A. The Amino-Acid Sequence and Carbohydrate Content of Phospholipase A2 from Bee Venom. Eur. J. Biochem. 1974, 48, 465-476.

16. Kuchler, K.; Gmachl, M.:Sippl, M. J.; Kreil, G. Analysis of the cDNA for Phospholipase A2 from Honeybee Venom Glands. The Deduced Amino Acid Sequence Reveals Homology to the Corresponding Vertebrate Enzymes. Eur. J. Biochem. 1989, 184, 249-254.

17. Hoffmann, D. R; Weimer, E. T.; Sakell, R. H.; Schmidt, M. Apis mellifera Venom Acid Phosphatase Precursor, mRNA, Complete cds, 2005: EMBL/GenBank/DDBJ databases, Brody School of Medicine, Greenville, NC 27834.

18. Schmidt, M.; Weimer, E. T.; Sakell, R. H.; Hoffman, D. R. Proteins in the High Molecular Weight Fraction of Honeybee Venom. J. Allergy Clin. Immunol. 2005, 115, 107.

19. Ownby, C. L.; Powell, J. R.; Jiang, M. S.; Fletcher, J. E. Melittin and Phospholipase A, from Bee (Apis mellifera) Venom Cause Necrosis of Murine Skeletal Muscle in Vivo. Toxicon 1997, 35, 67-80.

20. Kettner, A.; Hughes, G. J.; Frutiger, S.; Astori, M.; Roggero, M.; Spertini, F.; Corradin, G. J. Api m 6: A new venom allergen. J. Allergy. Clin. Immunol. 2001, 107, 914-920.

21. Hoffman, D. R. Hymenoptera Venom Allergens. Clin. Rev. Allergy Immunol. 2006, 30, 109-128.

22. Shkenderov, S. A Protease Inhibitor in Bee Venom. Identification, Partial Purification, and some Properties. FEBS Lett. 1973, 15, 343-347.

23. Kettner, A.; Henry, H.; Hughes, G. J.; Corradin, G.; Spertini, F. IgE and T-cell Responses to High-Molecular Weight Allergens from Bee Venom. Clin. Exp. Allergy 1999, 29, 394-401.

24. Winningham, K. M.; Schmidt, M.; Hoffman, D. R. Honeybee Venom Allergy: Cloning of the Apis mellifera Venom Protease. J. Allergy Clin. Immunol. 2001, 107, S221.

25. Winningham, K. M.; Fitch, C.; Schmidt, M.; Hoffman, D. Hymenoptera venom protease allergens. J. Allergy Clin. Immunol. 2004, 114, 928-933.

26. King, T. P.; Spangfort, M. D. Structure and Biology of Stinging Insect Venom Allergens. Int. Arch. Allergy Immunol. 2000, 123, 99-106.

27. Gmachl, M.; Kreil, G. The Precursors of the Bee Venom Constituents Apamin and MCD Peptide are Encoded by Two Genes in Tandem, Which Share the Same 3'-Exon. J. Biol. Chem. 1995, 270, 12704-12708.

28. Hoffman, D. R. Hymenoptera Venoms: Composition, Standardization, Stability. In Monograph of Insect Allergy 4th ed., Chap. IV, Levine, M. I.; Lockey, R. F. Eds.; American Academy of Allergy Asthma and Immunology: Milwaukee, 2003; p. 37-53.

29. King, T. P.; Guralnick, M. Hymenoptera Allergens. Clin. Allergy Immunol. 2004, 18, 339-353.

30. Golden, D. B. K. Insect Sting Allergy and Venom Immunotherapy: A Model and a Mystery. Curr. Rev. Allergy Clin. Immunol. 2005, 116, 464-465.

31. Freeman, T. M. Clinical Practice Hypersensitivity to hymenoptera stings. New. Engl. J. Med. 2004, 351, 1978-84.

32. Chaurand, P.; Schwartz, S. A.; Caprioli, R. M. Profiling and Imaging Proteins in Tissue Sections by MS. Anal. Chem. 2004, 76, 87A-93A.

33. Bunch, J.; Clench, M. R.; Richards, D. S. Determination of Pharmaceutical Compounds in Skin by Imaging Matrix-Assisted Laser Desorption/Ionization Mass Spectrometry. Rapid Commun. Mass Spectrom. 2004, 18, 3051-3060.

34. Trim, P. J.; Atkinson, S. J.; Princivalle, A. P.; Marshall, P. S.; West, A.; Clench, M. R. Matrix-Assisted Laser Desorption/Ionization Mass Spectrometry Imaging of Lipids in Rat Brain Tissue with Integrated Unsupervised Multivariant Statistical Analysis. Rapid Commun. Mass Spectrom. 2008, 22, 1503-1509.

35. Cornett, D. S.; Frappier, S. L.; Caprioli, R. M. MALDI-FTICR Imaging Mass Spectrometry of Drugs and Metabolites in Tissue. Anal. Chem. 2008, 80, 5648-5653.

36. Hoffman, D. R.; Shipman, W. H.; Babin, D. Allergens in Bee Venom II. Two New High Molecular Weight Allergenic Specificities. J. Allergy Clin. Immunol. 1977, 59, 147-153.

37. Hoffman, D. R. Allergens in Bee Venom III. Identification of Allergen B as an Acid Phosphatase. J. Allergy Clin. Immunol. 1977, 59, 364-366.

38. Ryssel, H.; Heitmann, C.; Germann, G.; Öhlbauer, M. Necrotizing Fasciitis After a Honey Bee Sting. Eur. J. Plast. Surg. 2007, 30, 11-14.

39. Larche, M. Peptide Immunotherapy. Immunol. Allergy Clin. North Am. 2006, 26, 321-332.

40. Habermann, E. Apamin. Pharmacol. Ther. 1984, 25, 255-270.

41. Hollander, T.; Aeed, P. A.; Elhammer, A. P. Characterization of the Oligosaccharide Structures on Bee Venom Phospholipase A2. Carb. Res. 1993, 247, 291-297. 